NASA Technical Memorandum 102524

ICOMP-90-06

\title{
Amplitude-Dependent Neutral Modes in Compressible Boundary Layer Flows
}

\section{J.S.B. Gajjar}

University of Exeter

Exeter, England

and Institute for Computational Mechanics in Propulsion

Lewis Research Center

Cleveland, Ohio

(NASA-TM-102524) AMPLITUNE-DEPFNDENT

NEUTRAL MDOES IN COMPRESSI SLE BOUNOARY LAYER

FLOWS (NASA) 19 P
CSCL 200 


\title{
AMPLITUDE-DEPENDENT NEUTRAL MODES IN COMPRESSIBLE \\ BOUNDARY LAYER FLOWS
}

\author{
J.S.B. Gajjar \\ Department of Mathematics \\ North Park Road \\ University of Exeter \\ Exeter EX4 4QE, England \\ and Institute for Computational Mechanics in Propulsion* \\ Lewis Research Center \\ Cleveland, Ohio 44135
}

\section{SUMMARY}

The ideas of Benney \& Bergeron (1969) and Davies (1970) on nonlinear critical layers are extended, and some new nonlinear neutral modes are computed for compressible boundary layer flow. A special case of the work is when the generalized inflexion point criterion holds. Neutral modes are found for a range of phase-speeds, dependent on the Mach number, and the properties of these are discussed. As in the linear case when the flow is relatively supersonic, multiple neutral modes exist. The behaviour of the neutral amplitude in some limiting cases is also considered, and it is found that the results are significantly different from that in incompressible flow when the flow is locally supersonic.

*Work funded by Space Act Agreement C-990066-G. 


\section{Int roduction}

The resurgence of interest in high speed flows has stimulated further work in the area of compressible boundary layer flows. Most of the early work on the linear instability of compressible boundary layers was carried out by Lees \& Lin (1946), Lees \& Reshotko (1960), Reshotko (1960) and this work and subsequent contributions are described in the reviews by Reshotko (1976), Mack (1984,1986). More recent work based on more formal asymptotic techniques includes that of Smith (1989), Gajjar \& Cole (1989a), Cowley \& Hall (1988) for boundary layers, Goldstein \& Leib (1989) for shear layers, as well as several other related papers dealing with various aspects of the numerical solution of the stability equations, see for example Malik (1987), and Jackson \& Grosch (1989). It is difficult to conduct experiments on high speed flows and to date there are very few reliable results and experimental data for transition in supersonic and hypersonic boundary layers. Not much work has been done to address the nonlinear instability of compressible boundary layers, theoretical, experimental or even numerical.

The current work forms a continuation of some earlier work described in Gajjar \& Cole (1989a) but with the emphasis now on studying the effect of a strongly nonlinear critical layer on the stability on a compressible boundary layer. The initial ideas on strongly nonlinear critical layers were first put forward by Benney \& Bergeron (1969) and Davies (1970), hereafter referred to as BBD, and were subsequently applied and extended by several authors. An excellent account of much of this work is given by Stewartson (1981). The underlying key ideas may be summarized as follows. If one considers the linear inviscid instability of an incompressible parallel fluid flow, say $U_{B}(Y)$, to normal mode disturbances proportional to $\delta e^{i \alpha(x-c t)}$, then the governing equation for instability is Rayleigh's equation. Here $Y$ is the normal coordinate, $x$ the streamwise coordinate, $t$ the time, $\alpha, c$, the wavenumber and phase-speed of the disturbance wave respectively, and $\delta$ is the small disturbance amplitude. If the base flow is taken to be Plane Poiseuille, or Blasius flow with the parallel flow approximation, then there are no unstable linear inviscid modes. For neutral modes, since Rayleigh's equation has a regular singularity at the critical point $Y=Y_{c}$ where $U_{B}\left(Y_{c}\right)=c$, the jump in the Reynolds stress across the critical point has to be zero. This jump however is also proportional to the jump in the 
logarithmic term arising in the local Frobenius expansion, across the critical point. The structure of the upper-branch neutral Tollmein-Schlichting mode for the flows mentioned above is equivalent to considering long wave limits of the Rayleigh equation with the inclusion of small viscous effects in appropriate thin regions. For these linear modes this jump is nonzero. However BBD postulated some new nonlinear neutral modes in which this jump was zero, and gave a description of the structure of this strongly nonlinear critical layer. The case of weaker nonlinearity was later considered by Haberman (1972) and he showed how the gap between the linear long wave upper-branch neutral modes and the BBD modes, could be bridged by setting up a structure in which the jump in the logarithmic term varied from its linear nonzero value, to zero for the BBD case. This jump depends on the size of the disturbance amplitude $\delta$. The various shortcomings and modifications of the early work are described in Stewartson (1981). Also many of the key ideas were later repeated/reformulated by Smith \& Bodonyi (1982), and Bodonyi et al. (1983), and they showed how this work could be put into a more formal asymptotic context as far as the stability of the flow was concerned.

For compressible boundary layer flows Gajjar \& Cole (1989a) (and hereafter referred to as GC), showed how the work of Haberman (1972), and Smith \& Bodonyi (1982) could be extended to include the effects of compressibility. One of the limits considered in this paper was the case when the jump in the logarithmic term approached zero corresponding to the strongly nonlinear critical layer. The present paper considers this latter problem and extends the work of Bodonyi, Smith \& Gajjar (1983), (and hereafter referred to as BSG), to compressible flows.

The role/importance of the nonlinear critical layer with respect to the stability of the flow, has been considered by the authors already cited. However a lot of this work has been based on the assumptions of neutral or equilibrium states only. The conclusions arising from these studies are thus limited and can only be interpreted as giving a qualitative description of the downstream development of a disturbance travelling wave. These studies suggest a shortening of scales and the outward movement of the critical layer from the wall into the free stream with increased disturbance amplitudes. The important issue of whether such states are stable or not cannot be deduced from this type of approach. The papers 
by (iajjar \& Smith (1985), Goldstein et al. (1987), for boundary layers, and Goldstein \& Hultgren (1988), Goldstein \& Leib (1989) together with many other similar papers, for shear layers, have attempted to address this very issue. From these studies it is apparent that the neutral states considered previously are unstable in some cases, although for shear layers saturated states do exist for some parameter ranges. Also despite many similarities in the disturbance structures and solutions, there are one or two subtle differences which can be important, see Goldstein \& Hultgren (1988).

In the present paper we concern ourselves with neutral states only. How relevant the work is is an open question since the conclusions from studies of incompressible flow may not necessarily carry over to those for compressible flow. In addition it is anticipated that the ideas and structure of the nonlinear compressible critical layer studied here will hold for the more general problem. Work on the full problem of following the spatial/temporal development of an unstable mode is in progress.

In section 2 below the basic problem for the strongly nonlinear critical layer is formulated. Section 3 describes the results and conclusions. Much of the detailed analysis and implications of of the critical layer structure and the solutions is omitted here. A fuller account of the work may be found in Gajjar \& Cole (1989b). 


\section{Problem Formulation}

We adopt the same notation and nondimensionalization used in $\mathrm{GC}$ so that $(x, y, t)$ are the nondimensional cartesian coordinates and time, $(u, v)$ the corresponding velocities, $(p, \rho, T)$ the pressure, density and temperature respectively. The local Mach number is $M_{\infty}$, and $R e$ is the Reynolds number which is assumed to be large. We also introduce the small parameter $\epsilon=R e^{-1 / 12}$. The basic boundary layer flow is given by,

$$
(u, v, p, \rho, T)=\left(U_{B}, \epsilon^{6} V_{B}, P_{B}, \rho_{B}, T_{B}\right)+\ldots
$$

and we assume that Chapman's viscosity law holds.

The disturbance structure for the flow is as sketched in Fig. 1 see also BSG, with I denoting the mainly inviscid region of thickness $O\left(\epsilon^{6}\right)$, and $Y=\epsilon^{-6} y$, is the boundary layer coordinate. In this region the flow quantities have expansions of the form:

$$
\begin{aligned}
u & =U_{B}(x, Y)+\epsilon u_{1 M}+\epsilon^{2} u_{2}+\ldots+\epsilon^{5} u_{5}+\ldots, \\
v & =\epsilon^{2} v_{2}+\ldots+\epsilon^{5} v_{5}+\ldots, \\
p & =p_{B}+\epsilon p_{1 M}+\epsilon^{2}\left(p_{2}+p_{2 M}\right)+\ldots+\epsilon^{5} p_{5}+\ldots, \\
\rho & =\rho_{B}(x, Y)+\epsilon \rho_{1 M}+\epsilon^{2} \rho_{2}+\ldots++\epsilon^{5} \rho_{5}+\ldots, \\
T & =T_{B}(x, Y)+\epsilon \theta_{1 M}+\epsilon^{2} \theta_{2}+\ldots+\epsilon^{5} \theta_{5}+\ldots
\end{aligned}
$$

The terms with suffices $M$ denote mean flow terms which arise because of the properties of the nonlinear critical layer. The fundamental travelling wave disturbances have amplitudes $O\left(\epsilon^{2}\right)$ and we write

$$
\left(u_{2}, v_{2}, p_{2}, \rho_{2}, \theta_{2}\right)=\left(U_{2}(Y) C, V_{2}(Y) S, P_{2}(Y) C, \bar{\rho}_{2}(Y) S, \Theta_{2}(Y) S\right)
$$

with $C=\cos X, S=\sin X$, and where

$$
\frac{\partial}{\partial x} \rightarrow \epsilon^{-6} \alpha_{0} \frac{\partial}{\partial X}+\frac{\partial}{\partial x}, \quad \frac{\partial}{\partial t} \rightarrow-\epsilon^{-6} \alpha_{0} c_{0} \frac{\partial}{\partial X} .
$$

The wavenumber $\alpha_{0}$ and the phase speed $c_{0}$ are taken to be real. After substitution into the Navier-Stokes equations the equation governing the stability of the flow is given by,

$$
L_{R} V_{2}=0
$$


where

$$
L_{R}=\frac{\partial}{\partial Y}\left(\xi \frac{\partial}{\partial Y}\right)-\left(q+\alpha_{0}^{2} \rho_{B}\right)
$$

with

$$
\xi=\frac{\rho_{B}}{1-\bar{M}^{2}}, \quad q=\frac{\partial}{\partial Y}\left(\xi \frac{\partial U_{B}}{\partial Y}\right) \frac{1}{\left(U_{B}-c_{0}\right)}, \quad \bar{M}^{2}=M_{\infty}^{2} \rho_{B}\left(U_{B}-c_{0}\right)^{2},
$$

is the compressible inviscid linear stability operator. The problem (2.2) has to be solved subject to the boundary conditions,

$$
V_{2}(0)=0, \quad \text { and } \quad V_{2}(\infty)<\infty
$$

for no slip at the wall and boundedness in the freestream, together with suitable jump conditions across the critical point $Y=Y_{c}$ where $U_{B}\left(Y_{c}\right)=c_{0}$. This point is a regular singular point of the differential equation and a Frobenius expansion locally shows that

$$
V_{2}(Y)=D_{0} \sum_{0}^{\infty} g_{n}\left(Y-Y_{c}\right)^{n}+D_{1}\left[\sum_{0}^{\infty} G_{n}\left(Y-Y_{c}\right)^{n}+\lambda\left(\sum_{0}^{\infty} g_{n}\left(Y-Y_{c}\right)^{n}\right) \ln \left(Y-Y_{c}\right)\right] \text {, }
$$

where

$$
\begin{gathered}
g_{0}=0, \quad g_{1}=1, \quad G_{0}=1, \quad G_{1}=0, \\
\lambda=\frac{\frac{\frac{\partial}{\partial Y}\left(\rho_{B} \frac{\partial U_{B}}{\partial Y^{Y}}\right)}{\rho_{B} \frac{\partial U_{B}}{\partial Y}}}{\text { at } Y=Y_{c},}
\end{gathered}
$$

and $D_{0}, D_{1}$ are arbitrary constants. To complete the specification of the problem the jump conditions across $Y=Y_{c}$ are necessary. Following the discussion in the introduction the appropriate jump conditions for the strongly nonlinear critical layer are that,

$$
\ln \left(Y-Y_{c}\right) \rightarrow \ln \left|Y-Y_{c}\right| \quad \text { for } \quad Y<Y_{c} .
$$

The amplitude equation which determines the possible neutral states can be derived as in BSG (1983) with suitable modifications to allow for compressibility. This gives the equation:

$$
R_{0} \mu_{c}\left(b_{1}\right)^{\frac{1}{2}}\left(\frac{R_{1}}{R_{0}}+\frac{2 b_{2}}{b_{1}}\right) \bar{C}^{(1)}=\frac{A^{\frac{3}{2}} g^{\prime}(0)^{2} c_{0}}{\alpha_{0}\left(T_{W}-M_{\infty}^{2} c_{0}^{2}\right)^{2}}\left(\frac{\mu_{W}}{\rho_{W}^{3} 2 c_{0}^{3}}\right)^{\frac{1}{2}}
$$

where

$$
b_{1}=\left.\frac{\partial U_{B}}{\partial Y}\right|_{Y_{c}}, \quad 2 b_{2}=\left.\frac{\partial^{2} U_{B}}{\partial Y^{2}}\right|_{Y_{c}}, \quad R_{1}=\left.\frac{\partial \rho_{B}}{\partial Y}\right|_{Y_{c}}, \quad T_{W}=T_{B}(Y=0)=\frac{1}{\rho_{W}},
$$




$$
\begin{gathered}
R_{0}=\left.\rho_{B}\right|_{Y_{r}}, \quad \bar{C}^{(1)}=\left[C^{(1)}(1-2 J)+2 C^{(2)} J-2 \pi J,\right. \\
J=\frac{R_{1} b_{1}}{\left(R_{1} b_{1}+2 b_{2} R_{0}\right)},
\end{gathered}
$$

and $\mu_{c}, \quad \mu_{W}$, are the coefficients of viscosity at the critical point and the wall respectively. Also the normalization $V_{2}=A g(Y)$ with $g\left(Y_{c}\right)=1$ has been used, $A$ being a scaled amplitude, and $C^{(1)}, C^{(2)}$, are the constants appearing in GC equation (4.8). The detailed analysis leading to $(2.2),(2.3)$, which requires consideration of the higher order terms in (2.1) and details of the nonlinear critical layer, and the wall layer, is given in Gajjar \& Cole (1989b). In summary the eigenvalue problem (2.2) determines $\alpha_{0}$ as a function of the phase-speed $c_{0}$ and the Mach number $M_{\infty}$, and the corresponding neutral amplitude is obtained from (2.3). The scaled amplitude used in BSG differs from that used here but is in fact equal to $P_{2}(0)$ and related to $A$ by,

$$
P_{2}(0)=\frac{c_{0} A g^{\prime}(0)}{\alpha_{0}\left(T_{W}-M_{\infty}^{2} c_{0}^{2}\right)} .
$$

For (2.3) to hold we require in addition that,

$$
\left(\frac{R_{1}}{R_{0}}+\frac{2 b_{2}}{b_{1}}\right) \bar{C}^{(1)}>0
$$

A special case of the above is when the generalized inflexion point criterion holds, namely $\lambda=0$. This gives the same wavenumber $\alpha_{0}=\alpha$, and phase-speed $c_{0}=c_{8}$ as in the linear case, but with the nonlinear neutral amplitude from (2.3).

For a critical layer to exist in the main part of the boundary layer we require $0<c_{0}<$ 1. Also we restrict ourselves to modes which are subsonic in the freestream (and then $V_{2}$ decays exponentially for large $Y$ ), so that, $1-1 / M_{\infty}<c_{0}<1$.

The basic flow for which most of the results were obtained is the flat plate boundary layer flow given by:

$$
\begin{gathered}
U_{B}=f^{\prime}(\eta), \quad f^{\prime \prime \prime}+f f^{\prime \prime}=0, \quad f(0)=f^{\prime}(0)=0, \quad f^{\prime}(\infty)=1, \\
T_{B}=1+\frac{(\gamma-1)}{2} M_{\infty}^{2}\left(1-f^{\prime 2}\right), \quad \text { for insulated walls, } \\
T_{B}=1+\frac{(\gamma-1)}{2} M_{\infty}^{2}\left(U_{B}-U_{B}^{2}\right)-\left(1-T_{W}\right)\left(1-U_{B}\right), \quad \text { with heat transfer. }
\end{gathered}
$$


Here $\eta$ is the Dorodnitsyn-Howarth variable with,

$$
\left(2 C x_{0}\right)^{\frac{1}{2}} \eta=\int_{0}^{Y} \rho_{B} d Y,
$$

and $C$ is Chapman's constant.

The solution(s) of (2.2) were obtained numerically using a shooting technique supplemented by series expansions near $Y=Y_{c}$. It was found convenient to work in terms of the variable $\eta$. Various checks were made including those for $M_{\infty}=0$, and for values of $M_{\infty}$ and $c_{0}$ for which the generalized inflexion point criterion was satisfied. 


\section{Results and Discussion}

Some preliminary results obtained are shown in Figures (3-8) It is apparent that there are different behaviours depending on whether $M_{\infty}<1, M_{\infty}>1$ and whether the flow is relatively subsonic or supersonic in some parts of the flowfield. For convenience each of these cases is dealt with separately below for insulated walls and for when there is heat transfer.

(a) Insulated walls. If the flow is everywhere relatively subsonic then $\bar{M}^{2}<1$ and this gives $1-1 / M_{\infty}<c_{0}<c_{a}=\sqrt{\left(\frac{(\gamma-1)}{2}+\frac{1}{M_{x}^{2}}\right)}$, whereas if $\bar{M}^{2}>1$ then $c_{b}=\max \left(c_{a}, 1-1 / M_{\infty}\right)<c_{0}<1$, are the possible values of the phase-speeds. The curves depicting these are shown in Fig. 2 where the curve for $c=c_{s}$ is also included. Further restrictions on $c_{0}$ arise from (2.5). For $c_{0}>c_{d},\left(c_{d}\right.$ is the value of $c_{0}$ when $\left.\bar{C}^{(1)}=0,\right)(2.5)$ is satisfied.

(i) $M_{\infty}<1$. For this case there is only one neutral mode, the properties of which are similar to that of incompressible mode computed in BSG. (Incidentally we find that the wavenumbers shown in BSG for Blasius flow should be multiplied by a factor $\sqrt{2}$ ). Results for $M_{\infty}=0, .5$, and 1 are shown in Fig. 3. The lower limit for the phase-speed is $c_{0}=c_{d}$ from (2.5), and as $c_{0} \rightarrow c_{d}+$, the amplitude goes to zero and from (2.3),

$$
A \sim\left[\left(\frac{R_{1}}{R_{0}}+\frac{2 b_{2}}{b_{1}}\right) \bar{C}^{(1)}\right]^{\frac{2}{3}} .
$$

If $M_{\infty}$ is small also this then matches with the corresponding limit in Cole (1989). As $c_{0} \rightarrow 1-$ the neutral wavenumber and amplitude increase indefinitely, for fixed values of $M_{\infty}$. This limiting structure is similar to that in Smith et al. (1989) for the corresponding incompressible mode, and we find that

$$
\alpha_{0} \sim \frac{3}{4} \zeta_{c}, \quad A \sim\left(T_{W}-M_{\infty}^{2} c_{0}^{2}\right)^{\frac{4}{3}} e^{\frac{3}{2} \zeta_{c}^{2}}
$$

where $\zeta_{c}$ is the asymptotic location of the critical point given by,

$$
.332 \frac{e^{-\frac{1}{2}\left(\zeta_{c}-\eta_{1}\right)^{2}}}{\left(\zeta_{c}-\eta_{1}\right)^{2}}=\left(1-c_{0}\right)
$$


and $\eta_{1}=1.2167$.

(ii) $\vec{M}^{2}<1, \quad M_{\infty}>1$. Since the flow is everywhere subsonic there is only one neutral mode, but with phase-speeds lying in the restricted range $\max \left(c_{d}, 1-1 / M_{\infty}\right)<c_{0}<c_{a}$. Figure 4 shows the variation of the neutral wavenumber and the neutral amplitude, against phase-speed for $M_{\infty}=2.2$. As $c_{0}$ approaches $1-1 / M_{\infty}$, the wavenumber goes to zero. We find that

$$
\alpha_{0} \sim\left(1-\bar{M}_{\infty}^{2}\right)^{\frac{1}{2}}, \quad A \sim\left(1-\bar{M}_{\infty}^{2}\right)^{\frac{1}{3}}, \quad P_{2}(0) \sim\left(1-\bar{M}_{\infty}^{2}\right)^{-\frac{1}{6}}
$$

consistent with the numerical results. For $c_{0} \rightarrow c_{a}-$ the wavenumber and amplitude approach constant values the reason being that $g^{\prime}(0)$ approaches zero,

$$
g^{\prime}(0) \sim\left(T_{W}-M_{\infty}^{2} c_{0}^{2}\right)
$$

(iii) $\bar{M}^{2}>1$. The flow becomes relatively supersonic near the wall and the differential equation changes type. As in the linear case, Mack (1984), there exist infinitely many neutral modes. The first four were computed and are shown in Figure 5, together with the corresponding neutral amplitudes. It can be seen that for certain values of $c_{0}$, for fixed values of $M_{\infty}$, the modes after initially decreasing start to increase, and then switch to take on the characteristics of the next higher mode. This is also seen in Figures 6(a-c), which show the eigenfunctions for different values of $c_{0}$. The sudden change in character of one mode to the next higher mode can be explained as being due to the presence/superposition of two distinct modes, one say, the WKB mode, and the other the 'subsonic' mode mentioned in (i) above, as can be seen clearly in Fig. 6. An analysis incorporating these ideas gives the wavenumber implicitly as,

$$
\alpha_{0} \sim \alpha_{k}=\left(\left(k+\frac{1}{4}\right) \pi+2 \tan ^{-1}\left[\frac{F^{\prime}(0) \zeta_{c} \phi(0)}{\alpha_{k} \phi^{\prime}(0)}\right]\right) / F\left(Y_{a}\right), \quad(k=0,1, \ldots)
$$

where

$$
\bar{M}_{\infty}^{2}\left(Y_{a}\right)=1, \quad F(Y)=\int_{0}^{Y}\left|1-\bar{M}^{2}\right|^{\frac{1}{2}} d y .
$$

The first term in the bracket on the right in (3.2) is the WKB mode, and the second describes the additional contribution from the 'subsonic' mode. The contribution from the WKB mode alone in (3.2) is superimposed on the computed values in Figure $5 \mathrm{a}$ and the 
agreement is quite good. The change in character of the mode can be explained by the presence of the second term in which $\zeta_{c}$ is as in (3.1) the location of the critical point. The quantity $\phi$ comes from solving the reduced eigenvalue problem for the subsonic mode but with slightly changes boundary conditions. For $\zeta_{c} / \alpha_{k}<<1$, the second term does not contribute. As $\alpha_{k}$ decreases then at some critical values of $c_{0}$ we have $\zeta_{c} / \alpha_{k}=O(1)$, and then the second term gives a nonzero contribution and $\alpha_{k}$ increases. For $\zeta_{c} / \alpha_{k}>>1$ the right side of (3.2) is exactly the same as that for the $(k+1)$ th WKB mode.

The limiting form (3.2) can be used to give the appropriate behaviour of $A$, but this is not done here. We note that a special case of the above, corresponding to the generalized inflexion point, is when $c_{0}=c_{8}$ and $\alpha_{0}=\alpha_{8}$. Results for this case, for linear theory, similar to those described above have been obtained independently also by Cowley \& Hall (1989) (they actually considered the limit for $M_{\infty}>>1$ ), and Brown \& Smith (unpublished but mentioned by Cowley \& Hall ). Our results agree with those of Cowley \& Hall (1989) for $M_{\infty}>>1$ since from (2.4) $\alpha_{k}=O\left(1 / M_{\infty}^{2}\right)$. The linear results for the generalised inflexion point mode given in Mack (1984) are different from those obtained here. It is not clear what viscosity law Mack has used, and this may be one reason for the difference. We also note that we could not obtain the first peak in the plot of the wavenumber against Mach number as shown in Fig. (9.2) in Mack (1984) although the second peak near $M_{\infty}=4$ was obtained. We cannot explain this discrepancy, and in fact our results are entirely consistent with the analysis and asymptotes in GC and here, and also Cowley \& Hall (1989).

Finally for large $M_{\infty}$ the neutral modes are confined to a narrow range of phase-speeds close to the freestream value, as can be seen also from Fig. 2.

(B) Heat transfer Only a few calculations were carried for cases when there is heat transfer. Some typical results are shown in Figures 7,8. For fixed values of the wall temperature $T_{W}$, the results obtained are broadly similar to those described above for the insulated case. For small values of $c_{0}$ and $M_{\infty}<1$, the asymptotes given in GC apply, and the numerical results agreed well with these. For $\bar{M}^{2}>1$ the asymptote (3.2) still holds and a comparison with the computed values is shown in Figure 8a, for the WKB mode.

In summary, we have considered the compressible version of the strongly nonlinear critical layer of BBD. Some new neutral modes have been computed and these have different 
properties depending on whether the flow is relatively subsonic or supersonic in some regions. In the latter case there exist infinitely many neutral states. Which if any of these can be observed in practical situations or in full numerical computations, is an open question. The work could be extended further to consider the next stage when the various limits are approached or when $M_{\infty}>>1$, but in our opinion it would be more useful to follow the spatial/temporal evolution of a linear unstable mode into the nonlinear stages of evolution. This would then bring into play some of the ideas discussed here and also in $\mathrm{GC}$, on nonlinear critical layers, and address the more important issue of whether any of these neutral states are significant or not. Work on this is currently in progress.

\section{Acknowledgements}

Some of the work described here was completed while the author was a summer visitor at ICOMP, NASA Lewis Research Center in 1988. Thanks are due to Dr. S. J. Cowley for providing data on the generalized inflexion point modes for comparison purposes. 


\section{References}

Benney, D. J., Stud. Appl. Math. 48. (1969), 181.

Bodonyi, R. J., Smith, F. T., and Gajjar, J., I.M.A. Jour. Appl. Math. 30 , (1983), 1.

Cole, J. W., Thesis, Univ. of Exeter (1989) (in prep.).

Cowley, S. J., and Hall, P., ICASE Rept. No. 88-i2, (1989).

Davies, R. E., Jour. Fluid Mech. 36, (1969), 337.

Gajjar, J., and Smith, F. T., Jour. Fluid Mech. 157, (1985), 53.

Gajjar, J. S. B., and Cole, J. W., Theor. Comp. Fluid. Dyn. 1, (1989a), 105.

Gajjar, J. S. B., and Cole, J. W., in prep. (1989b).

Goldstein, M. E., Durbin, P. A., and Leib, S. J., Jour. Fluid Mech. 183, (1987), 325.

Goldstein, M. E., and Hultgren, L., Jour. Fluid Mech. 197, (1988), 295.

Goldstein, M. E., and Leib, S. J., Jour. Fluid Mech. 207, (1989), 73.

Haberman, R., Stud. Appl. Math. 51, (1972), 139.

Jackson, T. L., and Grosch, C. E., ICASE Rept. No. 88-33, (1988).

Lees, L., and Lin, C. C., NASA Tech. Note No. 1115, (1946).

Lees, L., and Reshotko, E., Jour. Fluid Mech. 12, (1962), 555.

Mack, L. M., AGARD Rept. No. 709, (1984).

Mack, L. M., Proc. of the Symp. on the "Stability of Spatially Varying and Time Dependent Flows" (eds D. L. Dwoyer and M. Y. Hussaini), Springer-Verlag (1986).

Malik, M. R., A.I.A.A. Pap. No. 87-1414, (1987).

Reshotko, E., NASA Tech. Note No. D1220, (1960).

Reshotko, E., Ann. Rev. Fluid Mech. 8, (1976), 311.

Smith, F. T., Jour. Fluid Mech. 198, (1989), 127.

Smith, F. T., and Bodonyi, R. J., Jour. Fluid Mech. 118, (1982), 165.

Smith, F. T., Doorly, D., and Rothmeyer, A., Jour. Fluid Mech. (to appear), (1989).

Stewartson, K., I.M.A. Jour. Appl. Math. 27, (1981), 133. 


\section{ORIGINAL PAGE IS \\ OF POOR QUALITY}

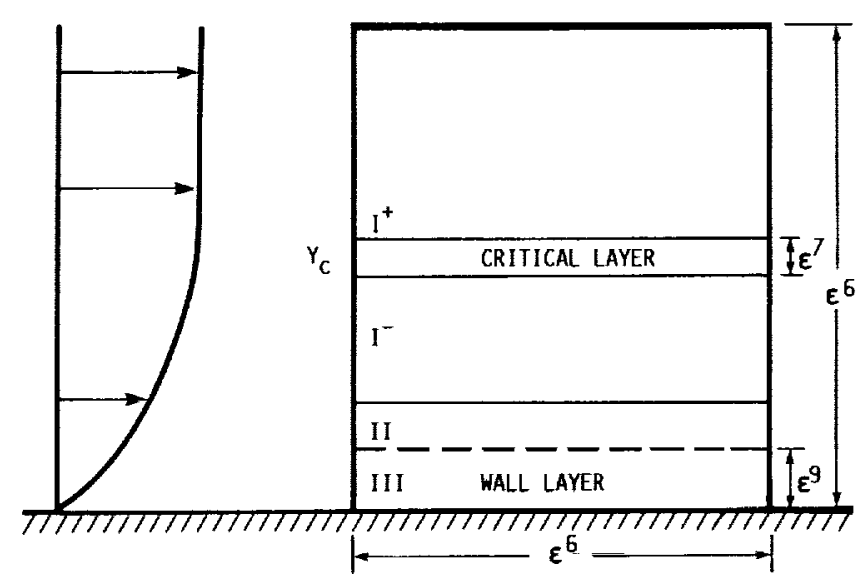

FIgURE 1. - NONLINEAR CRITICAL LAYER DISTURBANCE STRUCTURE.

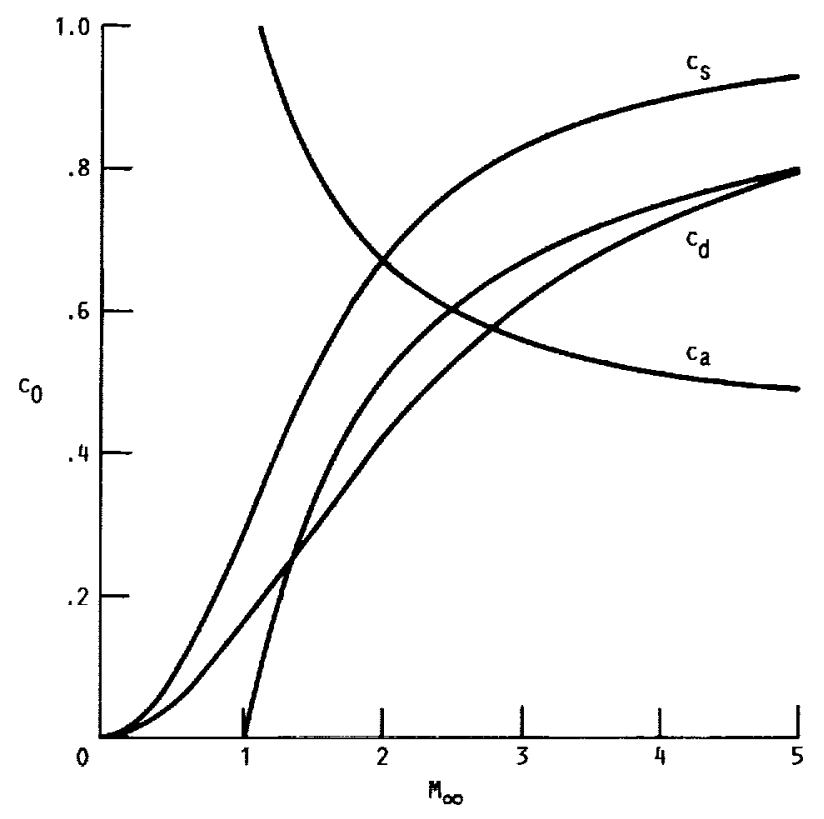

FIGURE 2. - PHASE-SPEEDS FOR BLASIUS FLOW WITH ADIABATIC HALL CONDITIONS: $c_{0}=c_{s}$ IS PHASE-SPEED FOR GENERALIZED INFLEXION POINT: $c_{0}=c_{a}$ IS PHASE-SPEED FOR WHICH $\tilde{m}^{2}=1$ AT THE WALL; $c_{0}=c_{d}$ IS CURVE FOR $\bar{c}^{(1)}=0$. THE UNLABELLED CURVE IS $c_{0}=1-1 / \mathrm{M}_{\infty}$.

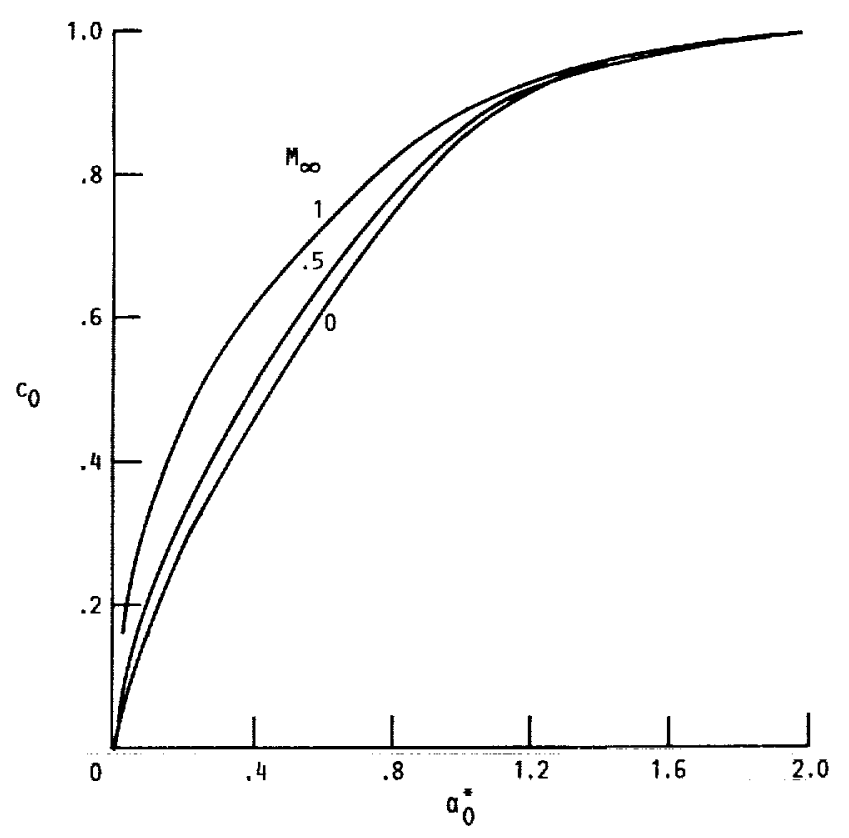

(a) MEUTRAL WAVENUMBER $a_{0}^{*}=a_{0}\left(2 x_{0} c\right)^{1 / 2}$ AGAinst PHASE-SPEED.

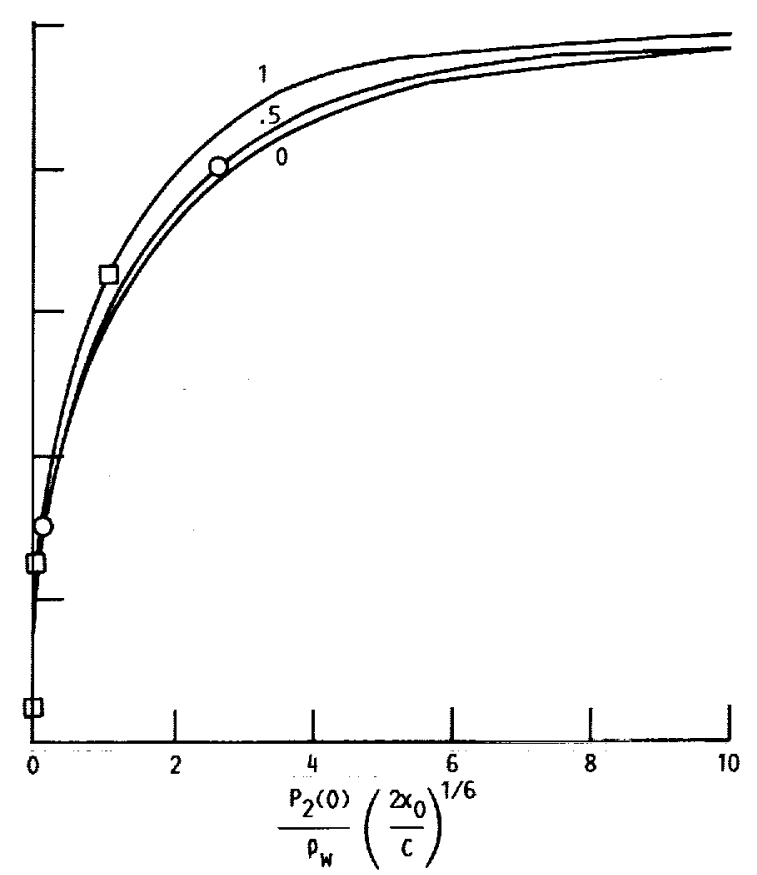

(b) CORRESPONDING NONLINEAR NEUTRAL AMPLITUDES.

FIGURE 3. - blasiUS FLOW WITH INSULATED WALL CONDITIONS FOR $M_{\infty}<1$. 

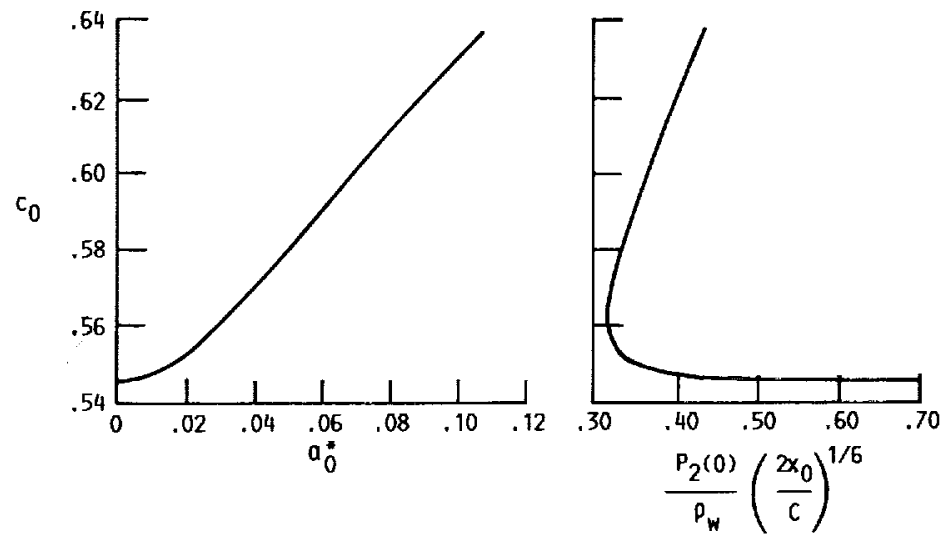

(a) NEUTRAL WAVENUMBER $a_{0}^{*}=a_{0}$ $\left(2 x_{0} c\right)^{1 / 2}$ AGAINST PHASE-SPEED.

(b) CORRESPONDING NONLINEAR NEUTRAL AMPLITUDES.

FIGURE 4. - BLASIUS FLOW WITH INSULATED WALL CONDITION FOR $M_{\infty}=2.2$.
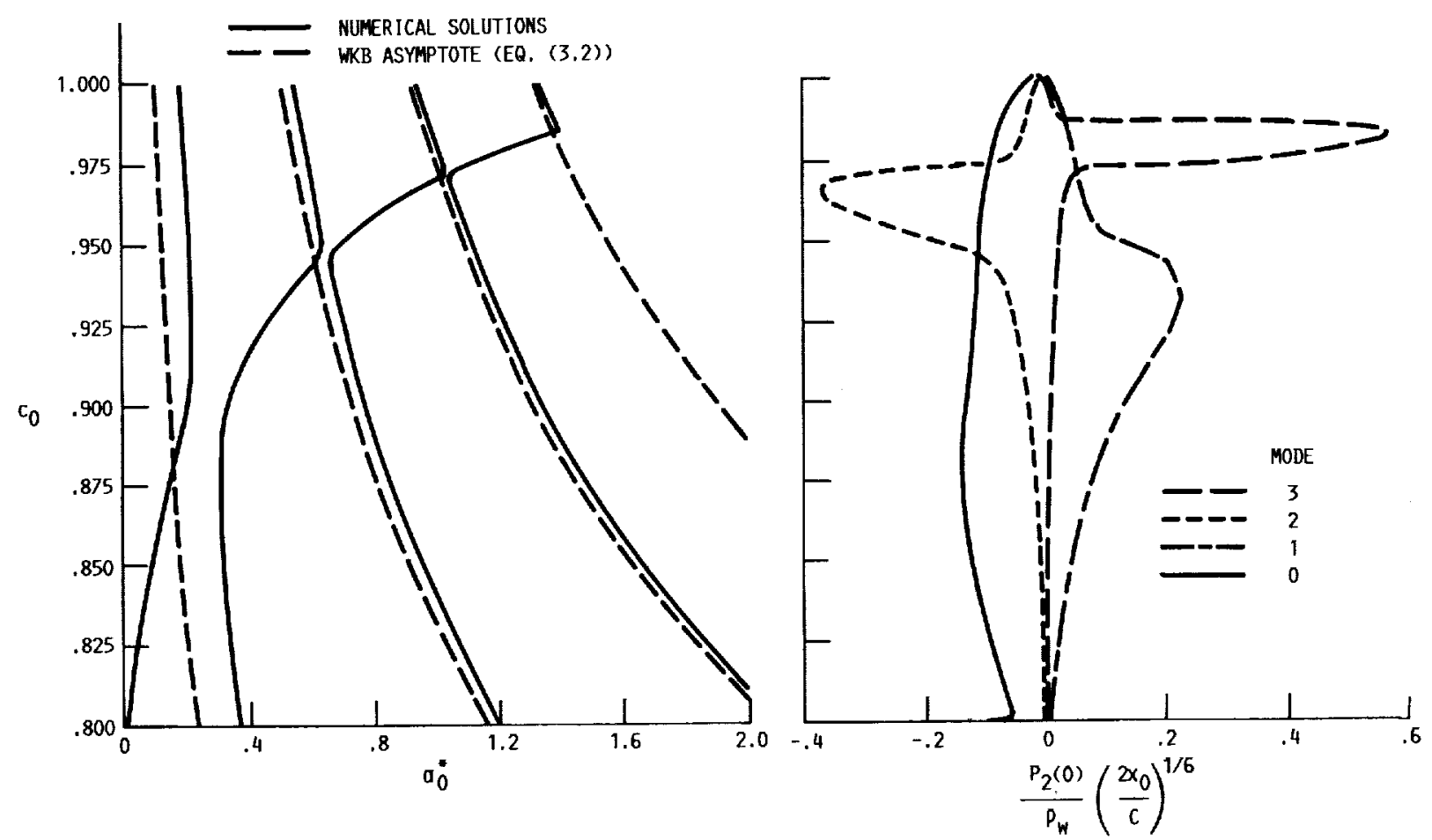

(a) NEUTRAL WAVENUMBER AGAINST PHASE-SPEED.

(b) CORRESPONDING NONLINEAR NEUTRAL AMPLITUDES FOR FIRST FOUR MODES.

FIGURE 5. - BLASIUS FLOW WITH INSULATED WALL CONDITIONS FOR $m_{\infty}=5$. 

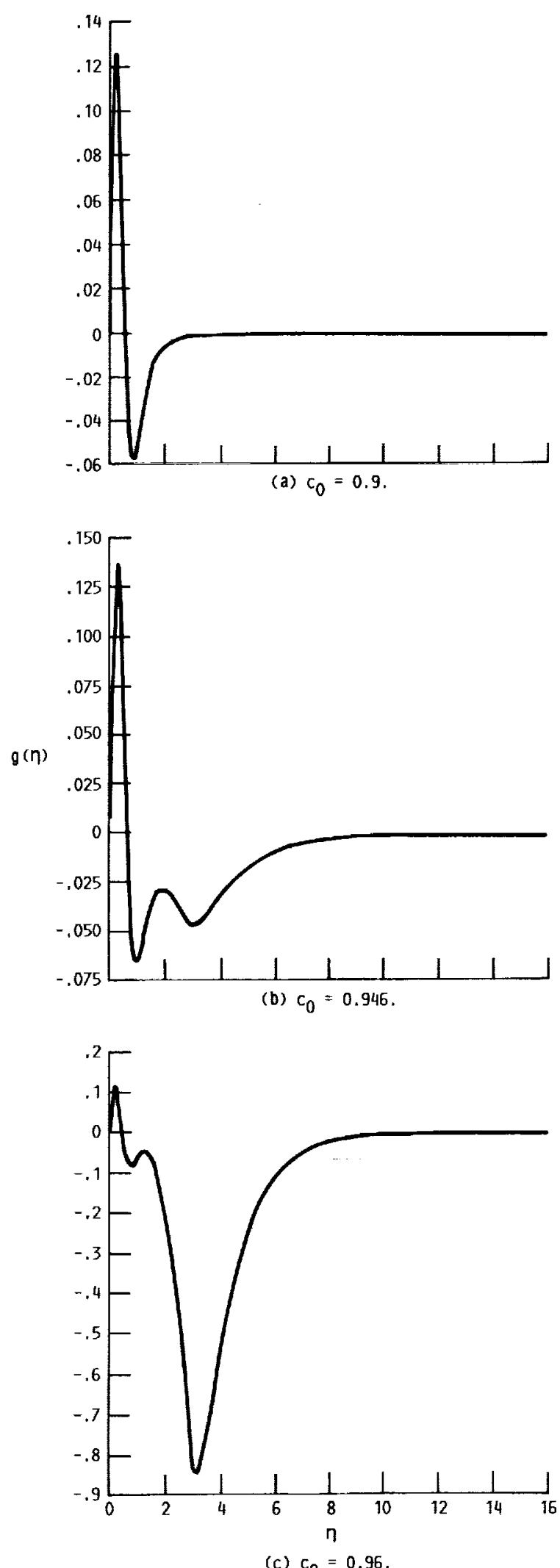

FIGURE 6. - EIGENFUNCTIONS FOR DIFFERENI PHASESPEEDS AND $M_{\infty}=5$ FOR BLASIUS FLOW WITH INSULATED WALL CONDITIONS. (THE NORMALIZATION $g$ ' $(0)=1$ HAS BEEN USED. ) 


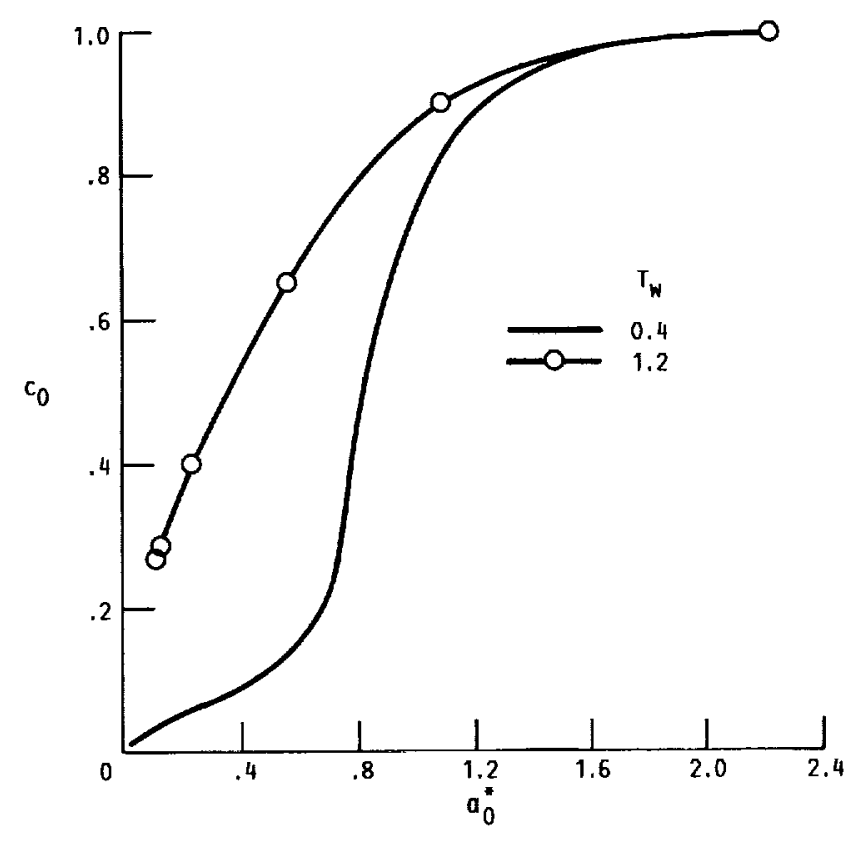

(a) NEUtral WaVENumBer AgAiNST PHASE-SPEED.

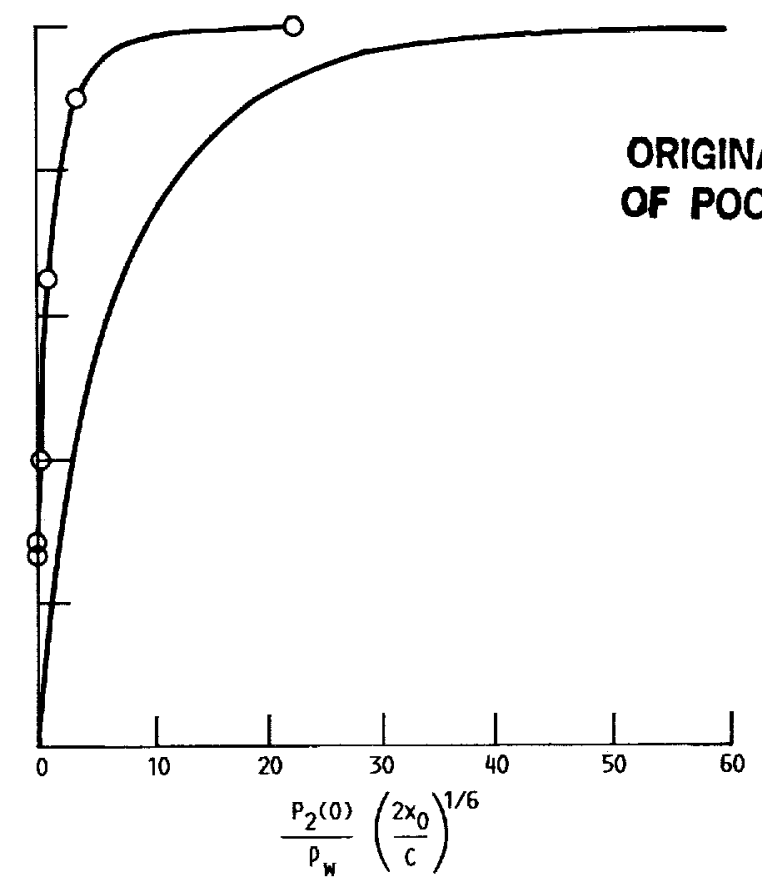

(b) CORRESPONDING NEUTRAL AMPLITUDE.

FIGURE 7. - BLASIUS FLOW WITH HEAT TRANSFER FOR $M_{\infty}=0.5$.

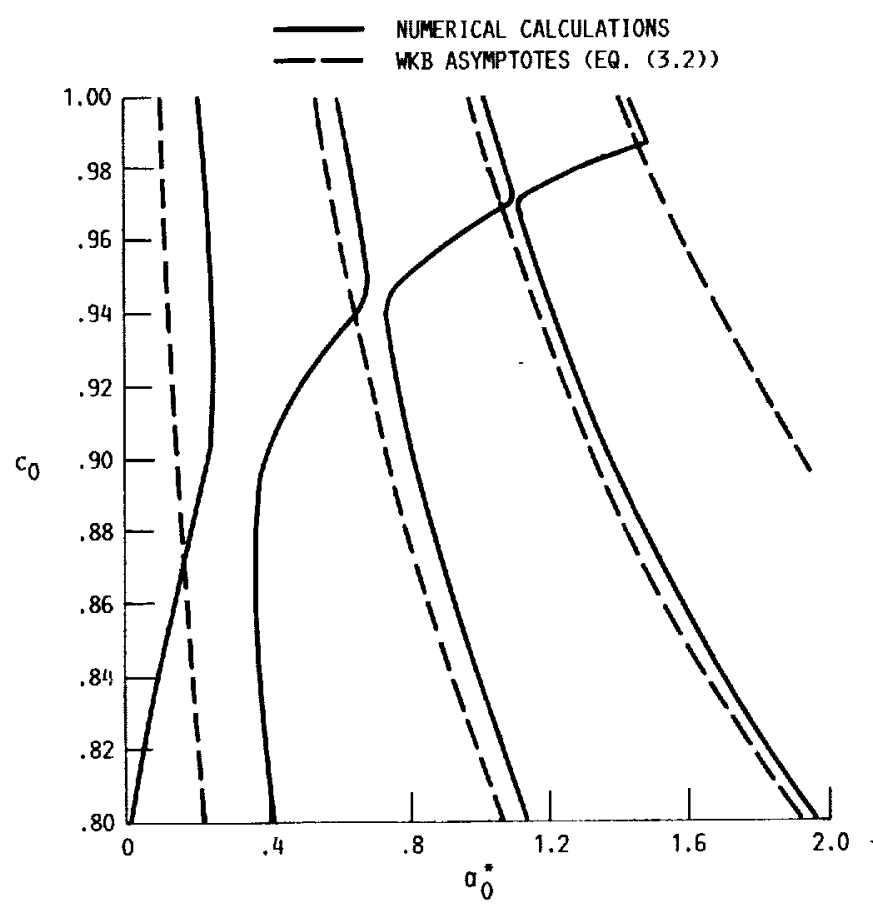

(a) NEUTRAL WAVENUHBER AGAINST PHASE-SPEED.

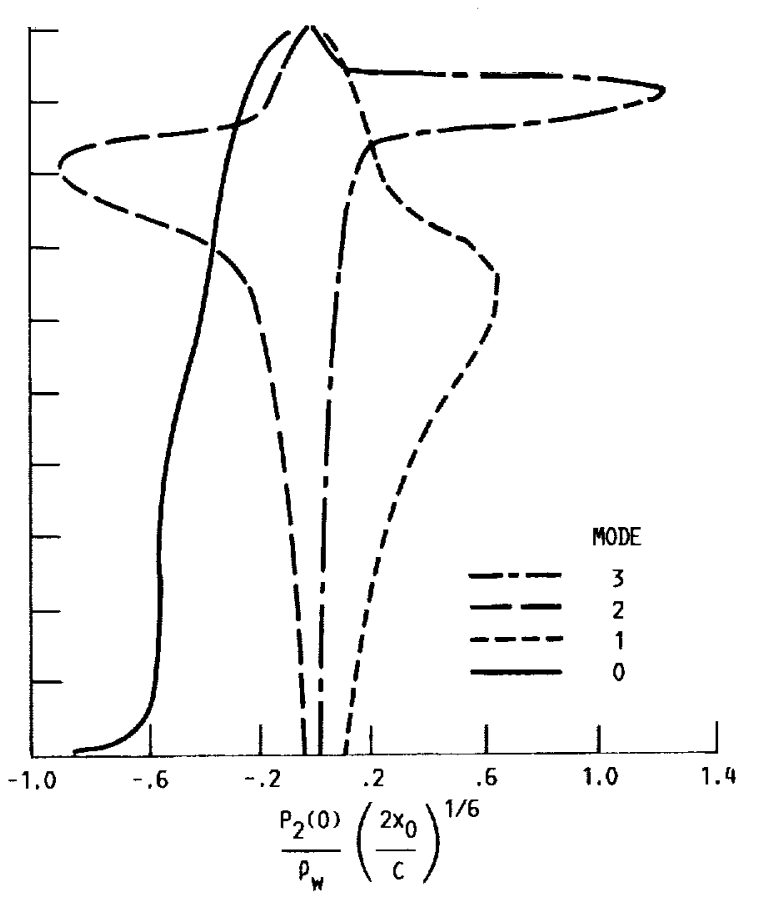

(b) CORRESPONDING NONL INEAR NEUTRAL AMPLITUDES FOR FIRST FOUR MODES.

FIGURE 8. - BLASIUS FLOW WITH HEAT TRANSFER FOR $M_{\infty}=5$ AND $T_{W}=3$. 


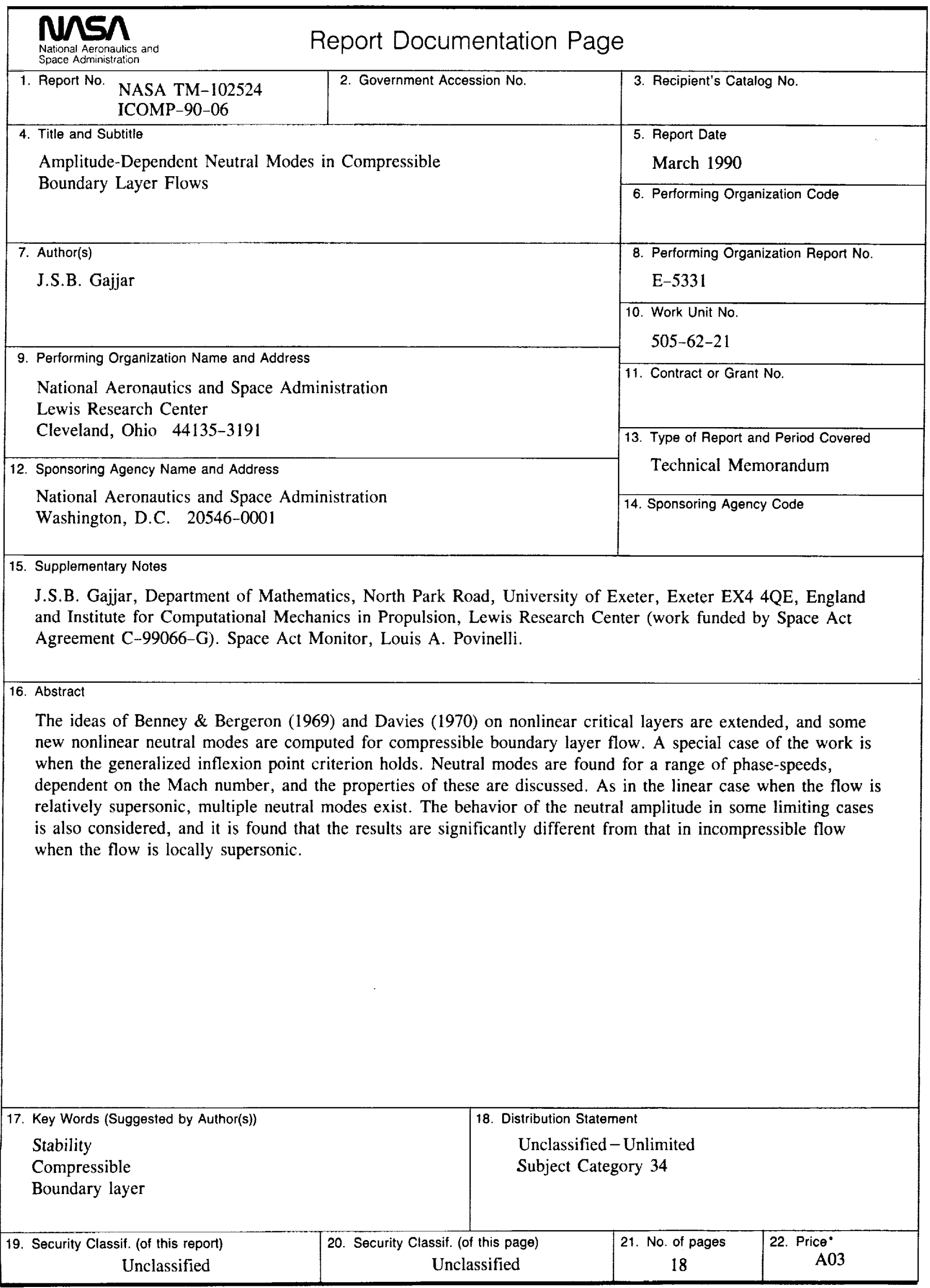

